\title{
Fast-convergent Fault Detection and Isolation in a Class of Nonlinear Uncertain Systems
}

\author{
Peng $\mathrm{Li}^{\mathrm{a}}$, Francesca Boem ${ }^{\mathrm{b}}$, Gilberto Pin ${ }^{\mathrm{c}}$ \\ ${ }^{a}$ School of Mechanical Engineering and Automation,Harbin Institute of Technology, \\ Shenzhen, China \\ ${ }^{b}$ Dept. of Electronic and Electrical Engineering, University College London, UK \\ ${ }^{c}$ Electrolux Italia S.p.A, Italy
}

\begin{abstract}
The present work proposes a fast-convergent fault detection and isolation (FDI) scheme for linear systems affected by model uncertainties, such as unknown inputs or unbounded nonlinearities. The finite-time convergence is attained by transforming the I/O signals through Volterra operators with suitably designed kernel functions. A novel feature of the proposed approach is the exploitation of a system decomposition that allows removing the effect of intractable uncertainties while recasting the system dynamics in a form applicable for Volterra operators to achieve non-asymptotic estimation. Remarkably, the proposed approach can reconstruct the state variables of the system in an arbitrarily short time and the fault can be diagnosed efficiently by imposing detection and isolation thresholds on transformed signals. The detectability and isolability of the fault are also characterized. The proposed FDI scheme is applied in simulation to a web process system to diagnose the presence of actuator faults. Simulation results confirm the effectiveness of the proposed scheme in two scenarios with nonlinear uncertainties.
\end{abstract}

Keywords: Non-asymptotic estimation, Volterra operator, Fault detection and isolation, Nonlinear uncertainty

\section{Introduction}

Fault detection and isolation (FDI) is a fundamental topic in modern engineering and has gained significant research attention. Classical approaches can be found in the books [1, 2, 3]. Model-based methodologies represent the pre5 ferred choice to design and implement FDI solutions. Based on the knowledge of the model, observers and estimators can be designed to reconstruct hidden state variables of the system and create auxiliary signals that permit to single out the faulty system from the healthy one. For linear systems, the Luenberger

Email addresses: lipeng2020@hit.edu.cn (Peng Li), f.boem@ucl.ac.uk (Francesca Boem), gilberto.pin@electrolux.it (Gilberto Pin)

Preprint submitted to European Journal of Control

July 7, 2020 
observer and the Kalman Filter are the most used tools to estimate the internal system's state and have been successfully implemented for fault detection and isolation (see [4, 5, 6]).

In recent years, considerable investigations have been devoted to enhancing the reliability of the FDI schemes, so to minimize the effects caused by the fault. More specifically, the effectiveness of many fault-tolerant control schemes

15 [2] rely on the correctness and promptness of the fault detection and isolation decisions (as discussed in [7]).

Furthermore, in many practical cases, the system may not be exactly known a-priori. Moreover, the system may present non-linearities which may not be properly dealt with by classical methodologies for linear systems. To address the 20 possible presence of non-linearities and uncertainties, a number of techniques have been proposed in the literature. Examples can be seen in [8, 9, 10] based on the adaptive approximation of the uncertain non-linearity in distributed systems, using, for example, radial basis function (RBF) methods. In this context, adaptive methods (see [11, 12]) and learning algorithms (as in [8]) can be applied 25 to identify the corresponding parametrization. Alternatively, the unknown input observer (UIO) is another powerful tool to eliminate the effect of the model uncertainty and to achieve the system monitoring goal which has been extensively used for FDI. For instance, in [13, a UIO is designed to detect the fault for multi-agent system networks, rendering the FDI scheme insensitive to model so uncertainty and non-linearities in the interconnections. The UIO has been implemented for FDI in many practical settings 14,15 .

All the estimation methods mentioned above for FDI are characterized by asymptotic convergence, which requires some time for the effects of the unknown initial conditions to decay. However, during the transient process, before con35 vergence, the sensitivity and reliability of the FDI methods could be reduced, thus possibly yielding delays in the detection of faults, misdetection or falsealarms. Therefore, fast or fixed-time estimation is desirable in FDI schemes. In the literature of fast-converging estimation, there are several methods and tools available. Sliding mode (SM) is among the most known methods to achieve 40 finite-time convergence [16, 17, 18. However, relying on discontinuous highgain injection, the SM methods may suffer from the chattering problem, that can be avoided by the use of higher-order SM [19, 20 and super twisting methods 21. Another class of finite-time estimation methodologies makes use of the integral tools, such as the well-known algebraic [22, 23. and modulating 45 function methods 24, 25, 26. However, univariate functions are used to modulate the integral in these two methods, which suffer from error accumulation and instability issues with expanding windows, as discussed in [27. To address these issues, as shown in the aforementioned works, extra efforts, such as sliding integral windows and periodically resetting are advised to avoid error accumu50 lation in the noisy scenario. On the contrary, a kind of kernel-based deadbeat estimation methodology with bivariate modulating functions inherently with internally stable integral calculation is proposed in 28]; here stability is inherently guaranteed so that no resetting, data memory nor moving window techniques are needed. Making use of the Volterra integral operator induced by suitably 
designed kernel functions, the kernel-based methodology removes the effect of the unknown initial conditions; in this way, no transient is required thus the convergence can be achieved in an arbitrarily short time. Paradigmatic frameworks have been proposed for parameter estimation [28, state estimation [29] and state-parameter joint estimation [30.

60 In this paper, a finite-time observer is designed to estimate the state of a class of uncertain systems in the presence of faults, with the capability of detecting and isolating the fault, again in finite-time. Based on the preliminary results in [31, where linear systems with bounded uncertainties are dealt with, a modified model is formulated to address the possible presence of unknown

65 model uncertainties, such as unknown inputs or unbounded non-linearities. A more comprehensive analysis including the detectability and isolability of the FDI scheme is considered herein. Indeed, by suitably decomposing the dynamics of the system, a fixed-time observer can be applied to eliminate the effects of the unknown initial conditions and the model uncertainties. As a result, finite70 time convergence of the state estimation can be attained to monitor the health status of the system online. The further application of Volterra operators to the estimated state variables allows the construction of auxiliary signals that are then used as fault indicators to detect and isolate the faults. Tuning rules for the kernel parameters of the Volterra operators are discussed. The model of

75 a web processing line is used as a simulation example to show the effectiveness of the proposed FDI scheme.

\section{Problem statement and system transformation}

Consider an uncertain multi-input-multi-output system modelled as:

$$
\mathcal{S}:\left\{\begin{array}{l}
\dot{x}(t)=A x(t)+B u(t)+E g_{U}(t, x, u, y)+F_{f} f(t, u, y) \\
y(t)=C x(t)
\end{array}\right.
$$

where $x(t) \in \mathbb{R}^{n}, u(t) \in \mathbb{R}^{m}$ and $y(t) \in \mathbb{R}^{q}$ are the state, the input and the output variables of the system respectively. $g_{U}(t, x, u, y) \in \mathbb{R}^{d}$ represents an unknown non-linear vectorial function, that could represent modelling uncertainty or an unknown input. $A, B, C, F_{f}$ are constant matrices with appropriate dimensions. $E \in \mathbb{R}^{p \times d}$ defines the effects of the unknown non-linearity to the system (1). The continuous function $f(t, u, y) \in \mathbb{R}^{p}$ models the effects of a general fault on the state dynamic equation. Similar with [32, 33, in this paper, we assume the possible faults are functions of time and I/O signals, including the measurable state variables . The fault function is modelled as

$$
f(t, u, y)=\mathcal{B}\left(t-T_{0}\right) \phi(t, u, y)
$$

where $\mathcal{B}\left(t-T_{0}\right)$ defines the fault time profile, which is equal to 0 before the unknown fault time $T_{0}$ and 1 after. The function $\phi(t, u, y) \in \mathbb{R}^{p}$ represents the 80 functional structure of the fault.

Our goal consists in designing a deadbeat observer from the measurement of the I/O signals, insensitive to the unknown non-linear function $g_{U}(t, x, u, y)$. 
Moreover, once the fault occurred at time $T_{0}$, it can be detected in a short period of time and the type of the fault can be isolated.

The following assumptions are made in this paper:

Assumption 1. $\operatorname{rank}(C E)=\operatorname{rank}(E)$.

This assumption is instrumental to guarantee the detectability of the fault from the system's output.

Assumption 2. For every complex number $\lambda$ with non-negative real part

$$
\operatorname{rank}\left[\begin{array}{cc}
A-\lambda I & E \\
C & 0
\end{array}\right]=n+\operatorname{rank}(E) .
$$

Referring to [34, Assumption 1 is equivalent to the existence of state and output transformations

$$
x(t)=T\left[\zeta_{1}^{\top}(t) \zeta_{2}^{\top}(t)\right]^{\top}, \quad y(t)=S\left[\eta_{1}^{\top}(t) \eta_{2}^{\top}(t)\right]^{\top}
$$

decomposing system (1) into two transformed linear systems having the following structure

$$
\begin{aligned}
& \mathcal{S}_{\zeta_{1}}:\left\{\begin{array}{l}
\dot{\zeta}_{1}(t)=A_{11} \zeta_{1}(t)+A_{12} \zeta_{2}(t)+B_{1} u(t)+E_{1} g_{U}(t, x, u, y) \\
\eta_{1}(t)=C_{11} \zeta_{1}(t)
\end{array}\right. \\
& \mathcal{S}_{\zeta_{2}}:\left\{\begin{array}{l}
\dot{\zeta}_{2}(t)=A_{21} \zeta_{1}(t)+A_{22} \zeta_{2}(t)+B_{2} u(t) \\
\eta_{2}(t)=C_{22} \zeta_{2}(t)
\end{array}\right.
\end{aligned}
$$

with

$$
\begin{array}{ll}
T^{-1} A T=\left[\begin{array}{ll}
A_{11} & A_{12} \\
A_{21} & A_{22}
\end{array}\right] & S^{-1} C T=\left[\begin{array}{cc}
C_{11} & 0 \\
0 & C_{22}
\end{array}\right] \\
T^{-1} B=\left[\begin{array}{ll}
B_{1}^{\top} & B_{2}^{\top}
\end{array}\right] & T^{-1} E=\left[\begin{array}{ll}
E_{1}^{\top} & 0
\end{array}\right],
\end{array}
$$

where $B_{1}$ and $C_{11}$ have the same number of rows with $B_{1}$ full row rank and

$90 \quad C_{11}$ invertible. Consequently, any complex number $\lambda$ that fails to satisfy Assumption 2, is an unobservable eigenvalue of the pair $\left(A_{22}, C_{22}\right)$. That is to say, as indicated in 34, Assumption 2 is equivalent to the detectability of the pair $\left(A_{22}, C_{22}\right)$. Meanwhile, $\zeta_{1}(t) \in \mathbb{R}^{p_{\star}}, p_{\star} \triangleq \operatorname{rank}(C E)$ and $\zeta_{2}(t) \in \mathbb{R}^{\left(n-p_{\star}\right)}$. Detailed calculation of the transformations, i.e. $(T, S)$ and properties can be 95 found in 34.

Notably, effects of the uncertainty $g_{U}(t, x, u, y)$ only appears on the dynamic of the first subsystem $\mathcal{S}_{\zeta_{1}}$. 


\section{Preliminaries: Volterra operator and non-asymptotic kernel}

To achieve finite-time fault detection and isolation of (2), Volterra operators and non-asymptotic kernel functions are the key tools of the proposed scheme. Basic concepts and algebra of the Volterra operators are briefly recalled for readers' convenience. Detailed mathematical features and characterization can be found in [29, 28, 35], and the reference therein.

Given a function $x(\cdot) \in \mathcal{L}_{l o c}^{2}\left(\mathbb{R}_{\geq 0}\right){ }^{1}$ its image through the Volterra operator $V_{K}$ induced by a Hilbert-Schmidt $\mathcal{H} \mathcal{S}$ Kernel Function $K(\cdot, \cdot): \mathbb{R} \times \mathbb{R} \rightarrow \mathbb{R}$ is usually denoted by $\left[V_{K} x\right](\cdot)$, and is defined by the inner product:

$$
\left[V_{K} x\right](t) \triangleq \int_{0}^{t} K(t, \tau) x(\tau) d \tau, \quad t \in \mathbb{R}_{\geq 0} .
$$

Lemma 3.1. [28] For a given $i \geq 0$, consider a signal defined as a function of time $x(t)$ and $x(\cdot) \in \mathcal{L}^{2}\left(\mathbb{R}_{\geq 0}\right)$ that admits the $i$-th derivative in $\mathbb{R}_{\geq 0}$ and a kernel function $K(\cdot, \cdot) \in \mathcal{H} \mathcal{S}$, having the $i$-th derivative with respect to the second argument, denoted as $K^{(i)}(t, \tau)$. After successive integral by parts, it holds that

$$
\begin{aligned}
{\left[V_{K} x^{(i)}\right](t)=} & \sum_{j=0}^{i-1}(-1)^{i-j-1} x^{(j)}(t) K^{(i-j-1)}(t, t) \\
& +\sum_{j=0}^{i-1}(-1)^{i-j} x^{(j)}(0) K^{(i-j-1)}(t, 0) \\
& +(-1)^{i}\left[V_{K^{(i)}} x\right](t),
\end{aligned}
$$

that is, the function $\left[V_{K} x^{(i)}\right](\cdot)$ is non-anticipative with respect to the lowerorder derivatives $x(\cdot), x^{(1)}(\cdot), \ldots, x^{(i-1)}(\cdot)$.

On the other hand, it is easy to tell from (3) that the effects of the derivatives can be manipulated by the kernel functions and their derivatives. As such, the following two types of kernel functions are significant in the proposed nonasymptotic estimation and FDI scheme.

Definition 3.1. If a kernel $K_{h}(\cdot, \cdot) \in \mathcal{H S}$ which is at least $(i-1)$-th order differentiable with respect to the second argument, verifies the condition

$$
K_{h}^{(j)}(t, 0)=0, \forall j \in\{0,1, \ldots, i-1\}
$$

then, it is called an $i$-th Order Bivariate Feedthrough Non-asymptotic Kernel $(B F-N K)[29]$.

\footnotetext{
${ }^{1} \mathcal{L}_{\text {loc }}^{2}\left(\mathbb{R}_{\geq 0}\right)$ denotes the Hilbert space of locally integrable function with domain $\mathbb{R}_{\geq 0}$ and range $\mathbb{R}$.
} 
A typical class of $N$-th order BF-NKs that we are using takes on the following form:

$$
K_{h}(t, \tau)=e^{-\omega_{h}(t-\tau)}\left(1-e^{-\bar{\omega} t}\right)^{N},
$$

tuned by parameters $\omega_{h} \in \mathbb{R}_{>0}$ and $\bar{\omega} \in \mathbb{R}_{>0}$.

As a result, induced by a BF-NK $K_{h}$, the Volterra image (3) reduces to

$$
\left[V_{K_{h}} x^{(i)}\right](t)=\sum_{j=0}^{i-1}(-1)^{i+j-1} x^{(j)}(t) K_{h}{ }^{(i-j-1)}(t, t)+(-1)^{i}\left[V_{K_{h}{ }^{(i)}} x\right](t),
$$

for all $i \in\{0, \ldots, N-1\}$

Definition 3.2. If a kernel $F(\cdot, \cdot) \in \mathcal{H S}$ which is at least $(i-1)$-th order differentiable with respect to the second argument, verifies the conditions

$$
F^{(j)}(t, 0)=0, F^{(j)}(t, t)=0, \forall j \in\{0,1, \ldots, i-1\},
$$

it is called an $i$-th Order Bivariate Causal Non-asymptotic Kernel (BC-NK)[28].

The typical shape of a 1-st order BC-NKs we are using is

$$
F(t, \tau) \triangleq e^{-\omega(t-\tau)}\left(1-e^{-\omega \tau}\right)\left[1-e^{-\omega(t-\tau)}\right],
$$

with the only tuning parameter $\omega$.

Recall (3), with BC-NK, the corresponding Volterra image (3) reduces to

$$
\left[V_{F} x^{(1)}\right](t)=-\left[V_{F^{(1)}} x\right](t) .
$$

\section{Deadbeat observer design and fault diagnosis scheme}

In this section, we design a deadbeat estimator based on the decomposed system (2). The estimator, exploiting the Volterra operator and the adoption of non-asymptotic kernel functions as in 29, is able to provide the state reconstruction of the non-linear system (1) non-asymptotically in arbitrary finite time.

\subsection{State reconstruction}

For system (1), with Assumptions 1 and 2, we denote

$$
T=\left[\begin{array}{ll}
T_{1} & T_{2}
\end{array}\right], \quad Q=S^{-1} \triangleq\left[\begin{array}{ll}
Q_{1}^{\top} & Q_{2}^{\top}
\end{array}\right]^{\top},
$$

where $T_{1} \in \mathbb{R}^{n \times p_{\star}}$ and $Q_{1} \in \mathbb{R}^{p_{\star} \times q}$. Thanks to the fact that $C_{11}$ is invertible, the estimates of $\zeta_{1}(t)$ can be retrieved directly from the measurement

$$
\hat{\zeta}_{1}(t)=C_{11}^{-1} Q_{1} y(t) .
$$


Similarly, $\eta_{2}(t)$ can be obtained by transforming the output $\hat{\eta}_{2}(t)=Q_{2} y(t)$. Recalling the fact that $\mathcal{S}_{\zeta_{2}}$ is detectable, a non-asymptotic state observer can be designed for system (2). For simplicity, in this paper, we assume that $\mathcal{S}_{\zeta_{2}}$ is a single-output system. Indeed, any observable system with multiple outputs can be reduced to a collection of single-output systems, using, for instance, the output counterpart of input reduction techniques in [36. The following linear transformation $P$ is introduced,

$$
P=M \mathcal{O}
$$

where $\mathcal{O}$ is the observability matrix of $\mathcal{S}_{\zeta_{2}}$ and $M$ is given as:

$$
\boldsymbol{M}=\left[\begin{array}{ccccc}
1 & 0 & 0 & \cdots & 0 \\
-a_{n_{\star}-1} & 1 & 0 & \ddots & \vdots \\
\vdots & \vdots & \ddots & \ddots & 0 \\
-a_{2} & -a_{3} & \cdots & 1 & 0 \\
-a_{1} & -a_{2} & \cdots & -a_{n_{\star}-1} & 1
\end{array}\right]
$$

where $n_{\star}=n-p_{\star}$ and $\left\{a_{i}, i \in\left\{0,1, \ldots, n_{\star}-1\right\}\right\}$ denotes the coefficients of the characteristic polynomial of the subsystems determined by the eigenvalues of matrix $A_{22}$.

As a result, with $z(t)=P \zeta_{2}(t), \mathcal{S}_{\zeta_{2}}$ can be rewritten in the observer canonical form:

$$
\left\{\begin{aligned}
\dot{z}(t) & =A_{c} z(t)+A_{c, 21} \zeta_{1}(t)+B_{c} u(t) \\
\eta_{2}(t) & =C_{c} z(t),
\end{aligned}\right.
$$

where $C_{c}=C_{22} P^{-1}=\left[\begin{array}{llll}1 & 0 & \ldots & 0\end{array}\right]$,

$$
A_{c}=P A_{22} P^{-1}=\left[\begin{array}{ccccc}
a_{n_{\star}-1} & 1 & 0 & \cdots & 0 \\
a_{n_{\star}-2} & 0 & 1 & \ddots & \vdots \\
\vdots & \vdots & \ddots & \ddots & 0 \\
a_{1} & 0 & \cdots & 0 & 1 \\
a_{0} & 0 & \cdots & 0 & 0
\end{array}\right] .
$$

Moreover, $A_{c, 21}=P A_{21} \triangleq\left[\alpha_{n_{\star}-1}^{\top}, \alpha_{n_{\star}-2}^{\top}, \ldots, \alpha_{0}^{\top}\right]^{\top}$ and

$$
B_{c}=P B_{2} \triangleq\left[\begin{array}{cccc}
b_{0, n_{\star}-1} & b_{0, n_{\star}-2} & \ldots & b_{0,0} \\
b_{1, n_{\star}-1} & b_{1, n_{\star}-2} & \ldots & b_{1,0} \\
& & & \\
b_{m-1, n_{\star}-1} & b_{m-1, n_{\star}-2} & \ldots & b_{m-1,0}
\end{array}\right]^{\top} \text {. }
$$


Let us consider the Volterra integral operator induced by BF-NK with $N \geq$ $n_{\star}$, so that the kernel is of at least $n_{\star}$-th order of non-asymptoticity.

Regarding to $14, \mathcal{S}_{\zeta_{2}}$ admits a corresponding Input/Output(I/O) realization

$$
\eta_{2}^{\left(n_{\star}\right)}(t)=\sum_{i=0}^{n_{\star}-1} a_{i} \eta_{2}^{(i)}(t)+\sum_{k=0}^{m-1} \sum_{i=0}^{n_{\star}-1} b_{k, i} u_{k}^{(i)}(t)+\sum_{i=0}^{n_{\star}-1} \alpha_{i} \zeta_{1, i}^{(i)}(t),
$$

where we denote $\zeta_{1}(t)=\left[\zeta_{1, n_{\star}-1}, \zeta_{1, n_{\star}-2}, \ldots, \zeta_{1,0}\right]^{\top}$.

Applying the Volterra operator induced by BF-NK to the system (15), recalling $(6)$ one can obtain

$$
\begin{gathered}
(-1)^{n_{\star}}\left[V_{K_{h}^{\left(n_{\star}\right)}} \eta_{2}\right](t)+\sum_{j=0}^{n_{\star}-1}(-1)^{n_{\star}+j-1} \eta_{2}^{(j)}(t) K_{h}^{\left(n_{\star}-j-1\right)}(t, t) \\
=\sum_{i=0}^{n_{\star}-1} a_{i}\left(\sum_{j=0}^{i-1}(-1)^{i+j-1} \eta_{2}^{(j)}(t) K_{h}^{(i-j-1)}(t, t)+(-1)^{i}\left[V_{K_{h}^{(i)}} \eta_{2}\right](t)\right) \\
+\sum_{k=0}^{m-1} \sum_{i=0}^{n_{\star}-1} b_{k, i}\left(\sum_{j=0}^{i-1}(-1)^{i+j-1} u_{k}^{(j)}(t) K_{h}^{(i-j-1)}(t, t)+(-1)^{i}\left[V_{K_{h}^{(i)}} u_{k}\right](t)\right) \\
+\sum_{i=0}^{n_{\star}-1} \alpha_{i}\left(\sum_{j=0}^{i-1}(-1)^{i+j-1} \zeta_{1, i}^{(j)}(t)+(-1)^{i}\left[V_{K_{h}}^{(j)} \zeta_{1, i}\right](t)\right)
\end{gathered}
$$

Note that for (14), the $r$-th state variable takes on the form

$z_{r}(t)=y^{(r)}(t)-\sum_{j=0}^{r-1} a_{n-r+j} y^{(j)}(t)-\sum_{k=0}^{m-1} \sum_{j=0}^{r-1} b_{k, n-r+j} u_{k}^{(j)}(t)-\sum_{j=0}^{r-1} \alpha_{n-r+j} \zeta_{1, j}^{(j)}(t)$,

for all $r \in\left\{0, \ldots, n_{\star}-1\right\}$. Consequently, with index rearrangement, 16 can be written as the following identity,

$$
\mu_{h}(t)=\gamma_{h}(t) z(t)
$$

where

$$
\begin{aligned}
\mu_{h}(t) \triangleq & (-1)^{n_{\star}-1}\left[V_{K_{h}\left(n_{\star}\right)} \eta_{2}\right](t)+\sum_{i=0}^{n_{\star}-1} a_{i}(-1)^{i}\left[V_{K_{h}^{(i)}} \eta_{2}\right](t) \\
& +\sum_{k=0}^{m-1} \sum_{i=0}^{n_{\star}-1}(-1)^{i} b_{k, i}\left[V_{K_{h}^{(i)}} u_{k}\right](t)+\sum_{i=0}^{n_{\star}-1}(-1)^{i} \alpha_{i}\left[V_{K_{h}^{(i)}} \zeta_{1, i}\right](t) \\
\gamma_{h}(t) \triangleq & {\left[(-1)^{n_{\star}-1} K_{h}^{\left(n_{\star}-1\right)}(t, t), \ldots, K_{h}(t, t)\right] . }
\end{aligned}
$$

Making use of $n_{\star}$ BF-NKs $K_{h}(t, \tau)$ tuned by different $\omega_{h}, h \in\left\{0, \ldots, n_{\star}-1\right\}$, the scalar equation(17) can be augmented into the matrix form

$$
\nu(t)=\Gamma(t) z(t)
$$


where $\nu(t)=\left[\mu_{0}(t), \mu_{1}(t), \ldots, \mu_{n_{\star}-1}(t)\right]^{\top}, \Gamma(t)=\left[\gamma_{0}(t)^{\top}, \gamma_{1}(t)^{\top}, \ldots, \gamma_{n_{\star}-1}(t)^{\top}\right]^{\top}$.

To streamline the notation, signals transformed by the application of the Volterra operators are denoted by $\left[V_{K_{h}^{(i)}} \kappa\right](t) \triangleq \xi_{\kappa, i, h}(t)$, where $\kappa$ represents signals $\eta_{2}, u_{k}$ and $\zeta_{1}$ respectively. Due to the specific structure of the kernel functions, the image functions can be computed as the output of a LTV system:

$$
\xi_{\kappa, i, h}^{(1)}(t)=-\omega_{h} \xi_{\kappa, i, h}+K_{h}^{(i)}(t, t) \kappa(t),
$$

with $\xi_{\kappa, i, h}(0)=0, \forall h \in\left\{0, \ldots, n_{\star}-1\right\}, i \in\left\{0, \ldots, n_{\star}\right\}$ for $\eta_{2}, i \in\left\{0, \ldots, n_{\star}-1\right\}$ for $u_{k}$ and $\zeta_{1}$.

Thanks to the specific shape of kernel defined in (5), the invertibility of $\Gamma(t)$ is guaranteed for any $t>0$ (strictly). Therefore, the state estimation of system (14) can be immediately obtained as

$$
\hat{z}(t)=\Gamma(t)^{-1} \nu(t), \quad \forall t>0
$$

Consequently, the state variables in $\mathcal{S}_{\zeta_{2}}$ can be retrieved as $\hat{\zeta}_{2}(t)=P^{-1} \hat{z}(t)$ and, thanks to (11), the estimated state vector of the original system can be computed by

$$
\hat{x}(t)=T_{1} \hat{\zeta}_{1}(t)+T_{2} \hat{\zeta}_{2}(t)
$$

Consequently, the state variables can be estimated with non-asymptotic convergence, i.e. $\exists T_{c} \in \mathbb{R}_{\geq 0}$

$$
\|\hat{x}(t)-x(t)\|=0, \forall t \geq T_{c} .
$$

\subsection{Fault detection and isolation}

Thanks to the fact that the state estimation enjoys a non-asymptotic convergence, the detection and isolation of the fault can be performed in a fast and accurate way.

Recall the process in (2), if a fault occurs after $T_{0}$, the system dynamics takes on the form

$$
\begin{aligned}
& \mathcal{S}_{\zeta_{1}}:\left\{\begin{array}{l}
\dot{\zeta}_{1}(t)=A_{11} \zeta_{1}(t)+A_{12} \zeta_{2}(t)+B_{1} u(t)+E_{1} g_{U}(t, x, u, y)+F_{f, 1} \phi(t, u, y) \\
\eta_{1}(t)=C_{11} \zeta_{1}(t)
\end{array}\right. \\
& \mathcal{S}_{\zeta_{2}}:\left\{\begin{array}{l}
\dot{\zeta}_{2}(t)=A_{21} \zeta_{1}(t)+A_{22} \zeta_{2}(t)+B_{2} u(t)+F_{f, 2} \phi(t, u, y) \\
\eta_{2}(t)=C_{22} \zeta_{2}(t)
\end{array}\right.
\end{aligned}
$$

where $\left[F_{f, 1}^{\top} F_{f, 2}^{\top}\right]^{\top} \triangleq T^{-1} F_{f}$. If $F_{f, 2}$ is full column rank, the fault signal verifies the following identity

$$
F_{f, 2} \phi(t, u, y)=\dot{\zeta}_{2}(t)-A_{22} \zeta_{2}(t)-A_{21} \zeta_{1}(t)-B_{2} u(t)
$$


where $\zeta_{1}(t)$ and $\zeta_{2}(t)$ can be exactly estimated while the derivative $\dot{\zeta}_{2}(t)$ becomes the main obstacle for detecting and identifying the fault signal promptly and accurately. Inspired by [37, the limited knowledge of the first derivative in (24) can be overcome by the Volterra operator with a 1-st order BC-NK, which gives

$$
F_{f, 2}\left[V_{F} f\right](t)=-\left[V_{F^{(1)}} \zeta_{2}\right](t)-A_{22}\left[V_{F} \zeta_{2}\right](t)-A_{21}\left[V_{F} \zeta_{1}\right](t)-B_{2}\left[V_{F} u\right](t)
$$

thanks to the feature of the Volterra operators (9).

Remarkably, the kernel function (8) can be rearranged as

$$
\begin{aligned}
F(t, \tau) & =F_{0,1}(t, \tau)+F_{0,2}(t, \tau) \\
F^{(1)}(t, \tau) & =F_{1,1}(t, \tau)+F_{1,2}(t, \tau),
\end{aligned}
$$

where

$$
\begin{array}{ll}
F_{0,1}(\tau)=\left(e^{\omega \tau}-1\right) e^{-\omega t}, & F_{0,2}(\tau)=\left(e^{\omega \tau}-e^{2 \omega \tau}\right) e^{-2 \omega t} \\
F_{1,1}(\tau)=\omega e^{\omega \tau} e^{-\omega t}, & F_{1,2}(\tau)=\left(\omega e^{\omega \tau}-2 \omega e^{2 \omega \tau}\right) e^{-2 \omega t} .
\end{array}
$$

As a result, the image functions $\chi_{\kappa, i}(t)=\left[V_{F^{(i)}} \kappa\right](t)$ can be calculated by an internally stable LTV system

$$
\left\{\begin{array}{l}
\varsigma_{\kappa, i}^{(1)}(t)=G \varsigma_{\kappa, i}(t)+E_{i}(t) \kappa(t) \\
\chi_{\kappa, i}(t)=H \varsigma_{\kappa, i}(t),
\end{array}\right.
$$

where $i=\{0,1\}$ and $\kappa$ represents for $\zeta_{1}$ and $\hat{\zeta}_{2}$

$$
\begin{aligned}
G & =\operatorname{diag}(-\omega,-2 \omega) \\
E_{i}(t) & =\left[F_{i, 1}(t, t), F_{i, 2}(t, t)\right]^{\top} \\
H & =\left[\begin{array}{ll}
1 & 1
\end{array}\right] .
\end{aligned}
$$

In this way, it is possible to estimate in a non-asymptotic way the transformed fault function. Even if the true fault function is not available, we will show that the FDI task can be achieved by exploiting such a transformed fault function. Indeed, a fault detection residual can be derived based on the transformed signals

$$
\begin{aligned}
r_{F D}(t) & \triangleq\left\|F_{f, 2}\left[V_{F} f\right](t)\right\| \\
& =\left\|-\left[V_{F^{(1)}} \zeta_{2}\right](t)-A_{22}\left[V_{F} \zeta_{2}\right](t)-A_{21}\left[V_{F} \zeta_{1}\right](t)-B_{2}\left[V_{F} u\right](t)\right\|,
\end{aligned}
$$

140 to indicate the health status of the system, where $\|\cdot\|$ denotes the Euclidean norm.

Fault detection decision A fault occurring to the system is detected by the proposed fault detection scheme at time $t=T_{D}$, assuming no noise is 
affecting the system, if the fault detection residual $r_{F D}$ is different from zero, i.e. $r_{F D}\left(T_{D}\right) \neq 0$.

After the detection of the fault, a fault isolation mechanism is activated by resetting the estimator (25), which means resetting all the transformations in 27). Correspondingly, we define a new notation to represent the operator after the resetting at $t=T_{D}$ :

$$
\left[\breve{V}_{F} \kappa\right](t) \triangleq \int_{0}^{t-T_{D}} F\left(t-T_{D}, \tau\right) \kappa\left(\tau+T_{D}\right) d \tau, \forall t \geq T_{D},
$$

where $\kappa$ can denote the signals $\zeta_{1}, \zeta_{2}, u$ and $\phi$ respectively.

For fault isolation, a set of faults $\mathcal{F}$ is assumed to contain all possible $N_{\mathcal{F}}$ types of fault functions $\phi_{i}(t, u, y), i \in\left\{0, \ldots, N_{\mathcal{F}}-1\right\}$, that can occur to the system.

Considering the system in the faulty mode (23), for each possible fault $\phi_{i}(t, x, y)$ one can obtain a fault isolation residual

$$
\begin{aligned}
& r_{F I, i}(t)=\left\|F_{f, 2}\left(\left[\breve{V}_{F} \phi\right](t)-\left[\breve{V}_{F} \phi_{i}\right](t)\right)\right\| \\
& =\left\|-\left[\breve{V}_{F^{(1)}} \zeta_{2}\right](t)-A_{22}\left[\breve{V}_{F} \zeta_{2}\right](t)-A_{21}\left[\breve{V}_{F} \zeta_{1}\right](t)-B_{2}\left[\breve{V}_{F} u\right](t)-F_{f, 2}\left[\breve{V}_{F} \phi_{i}\right](t)\right\|,
\end{aligned}
$$

Fault isolation By using the fault isolation residual (30), the $p$-th fault is excluded if $r_{F I, p}(t) \neq 0$. If there exists a $T_{I}>T_{D}$ such that all the faults are excluded but the $q$-th one, i.e. $r_{F I, q}(t)=0, \forall t>T_{I}$, then $q$-th fault is isolated at time $T_{I}$.

\section{Fault detection and isolation of systems with full order uncertainty}

Based on the previous FDI scheme, in this section, we are going to analyse the system with full order model uncertainty, i.e.

$$
\left\{\begin{aligned}
\dot{x}(t) & =A x(t)+B u(t)+g(t, x, u, y)+F_{f} f(t, u, y), \\
y_{d}(t) & =C x_{d}(t)
\end{aligned}\right.
$$

where $g(t, x, u, y)$ is a combination of uncertainties with known and unknown boundedness. We assume $g(t, x, u, y)$ can be divided into two parts, such that

$$
g(t, x, u, y)=g_{L}(t, x, u, y)+E g_{U}(t, x, u, y),
$$

where $g_{L}(t, x, u, y)$ is the part that can be bounded by a computable known bound i.e. $\left|g_{L}(t, x, u, y)\right| \leq \bar{g}_{L}(t) \in \mathcal{R}^{n}$ and $g_{U}(t, x, u, y)$ is the unknown uncertainties for which we do not know its boundedness. Notably, Assumption 1 , 2 are still necessary for the following discussion. 
Following the same transformation process in Section 4 , the nominal system (31) is rearranged as

$$
\begin{aligned}
& \mathcal{S}_{\zeta_{1}}:\left\{\begin{array}{l}
\dot{\zeta}_{1}(t)=A_{11} \zeta_{1}(t)+A_{12} \zeta_{2}(t)+B_{1} u(t)+E_{1} g_{U}(t, x, u, y)+g_{1}(t, x, u, y), \\
\eta_{1}(t)=C_{11} \zeta_{1}(t),
\end{array}\right. \\
& \mathcal{S}_{\zeta_{2}:}:\left\{\begin{array}{l}
\dot{\zeta}_{2}(t)=A_{21} \zeta_{1}(t)+A_{22} \zeta_{2}(t)+B_{2} u(t)+g_{2}(t, x, u, y), \\
\eta_{2}(t)=C_{22} \zeta_{2}(t),
\end{array}\right.
\end{aligned}
$$

where $\left[g_{1}(t, x, u, y)^{\top} g_{2}(t, x, u, y)^{\top}\right]^{\top} \triangleq T^{-1} g_{L}(t, x, u, y)$. As such, the $g_{1}(t, x, u, y)$ and $g_{2}(t, x, u, y)$ inherit the boundedness of $g_{L}(t, x, u, y)$ such that

$$
\left[\begin{array}{l}
\left|g_{1}(t, x, u, y)\right| \\
\left|g_{2}(t, x, u, y)\right|
\end{array}\right] \leq\left[\begin{array}{c}
\bar{g}_{1}(t) \\
\bar{g}_{2}(t)
\end{array}\right] \triangleq\left|T^{-1}\right| \bar{g}_{L}(t),
$$

where $|\cdot|$ denotes the component-wise absolute value.

Applying the same state observer scheme from (11) to 222, the estimates of the state variables are prone to be contaminated by $g_{L}(t, x, u, y)$. However, thanks to the proven Input-to-State Stable (I.S.S) property of the Volterra transformation with the BF-NK, a computable bound can be derived for the transformed signals, as in 35. In this context, an FDI scheme can be still designed to diagnose the fault by introducing proper thresholds.

Recalling (11), it is worth noting that the uncertain function $g_{1}(t, x, u, y)$ does not have influence on the estimates of state variables of $\mathcal{S}_{\zeta_{1}}$, thus we can write: $\hat{\zeta}_{1}(t)=\zeta_{1}(t)=C_{11}^{-1} Q_{1} y(t)$.

Considering the effect of $g_{2}(t, x, u, y) \triangleq\left[g_{2,0}(t, x, u, y), \ldots, g_{2, n_{\star}-1}(t, x, u, y)\right]^{\top}$ on the subsystem $\mathcal{S}_{\zeta_{2}}$ in 31 , making use of the observer (19), the estimated state variables take on the form

$$
\hat{\zeta}_{2}(t)=P^{-1} \Gamma(t)^{-1}\left[\hat{\mu}_{0}(t), \hat{\mu}_{1}(t), \ldots, \hat{\mu}_{n_{\star}-1}\right](t),
$$

where

$$
\begin{aligned}
\hat{\mu}_{h}(t)= & (-1)^{n_{\star}-1}\left[V_{K_{h}{ }^{\left(n_{\star}\right)}} \eta_{2}\right](t)+\sum_{i=0}^{n_{\star}-1} a_{i}(-1)^{i}\left[V_{K_{h}^{(i)}} \eta_{2}\right](t) \\
& +\sum_{k=0}^{m-1} \sum_{i=0}^{n_{\star}-1}(-1)^{i} b_{k, i}\left[V_{K_{h}^{(i)}} u_{k}\right](t)+\sum_{i=0}^{n_{\star}-1}(-1)^{i} \alpha_{i}\left[V_{K_{h}^{(i)}} \zeta_{1, i}\right](t),
\end{aligned}
$$


for all $h \in\left\{0, \ldots, n_{\star}-1\right\}$. In this case, the actual uncertain system verifies

$$
\begin{aligned}
\mu_{h}(t)= & (-1)^{n_{\star}-1}\left[V_{K_{h}\left(n_{\star}\right)} \eta_{2}\right](t)+\sum_{i=0}^{n_{\star}-1} a_{i}(-1)^{i}\left[V_{K_{h}^{(i)}} \eta_{2}\right](t) \\
& +\sum_{k=0}^{m-1} \sum_{i=0}^{n_{\star}-1}(-1)^{i} b_{k, i}\left[V_{K_{h}^{(i)}} u_{k}\right](t)+\sum_{i=0}^{n_{\star}-1}(-1)^{i} \alpha_{i}\left[V_{K_{h}^{(i)}} \zeta_{1, i}\right](t) \\
& +\sum_{i=0}^{n_{\star}-1}(-1)^{i}\left[V_{K^{(i)}} g_{2, n_{\star}-1-i}\right](t) .
\end{aligned}
$$

Therefore, the state estimation error takes on the form

$$
\epsilon_{\zeta_{2}}(t) \triangleq\left|\hat{\zeta}_{2}(t)-\zeta_{2}(t)\right|=\left|P^{-1} \Gamma(t)^{-1}\left[\epsilon_{\mu_{0}}(t), \epsilon_{\mu_{1}}(t), \ldots, \epsilon_{\mu_{n_{\star}-1}}(t)\right]^{\top}\right|
$$

where

$$
\epsilon_{\mu_{h}}(t) \triangleq \hat{\mu}_{h}(t)-\mu_{h}(t)=-\sum_{i=0}^{n_{\star}-1}(-1)^{i}\left[V_{K_{h}^{(i)}} g_{2, n_{\star}-1-i}\right](t) .
$$

Moreover, we notice that the BF-NK can be expressed as

$$
K_{h}^{(i)}(t, \tau)=e^{-\omega_{h} t} \sum_{q=0}^{n_{\star}}\left(\begin{array}{c}
n_{\star} \\
q
\end{array}\right)\left(\omega_{h}-q \bar{\omega}\right)^{i} e^{\left(\omega_{h}-q \bar{\omega}\right) \tau} .
$$

Therefore, signals transformed by a BF-NK Volterra operator admits the upper bound:

$$
\begin{aligned}
{\left[V_{K_{h}^{(i)}} g_{2, n_{\star}-1-i}\right](t) } & =e^{-\omega_{h} t} \sum_{q=0}^{n_{\star}}\left(\begin{array}{c}
n_{\star} \\
q
\end{array}\right)\left(\omega_{h}-q \bar{\omega}\right)^{i} \int_{0}^{t} e^{\left(\omega_{h}-q \bar{\omega}\right) \tau} g_{2, n_{\star}-1-i}(\tau) d \tau \\
& \leq \sum_{q=0}^{n_{\star}}\left(\begin{array}{c}
n_{\star} \\
q
\end{array}\right)\left(\omega_{h}-q \bar{\omega}\right)^{i}\left(e^{-q \bar{\omega} t}-e^{-\omega_{h} t}\right) \bar{g}_{2, n_{\star}-1-i} \\
& \triangleq \bar{\xi}_{g, i, h}
\end{aligned}
$$

170 where $\bar{g}_{2, j}$ denotes the $j$-th element of the bound signal $\bar{g}_{2}(t)$. Remarkably, $\omega_{h}$ and $\bar{\omega}$ can be properly tuned to tighten the bound $\bar{\xi}_{g, i, h}, \forall i \in\left\{0, \ldots, n_{\star}-\right.$ $1\}, \forall h \in\left\{0, \ldots, n_{\star}-1\right\}$.

Remark 5.1. The computation of the estimation error bound (34) at the beginning of the simulation could arouse numerical issues as $\Gamma(t)$ is nearly singular 175 at the initial time instants. As such $\left\|\Gamma(t)^{-1}\right\|$ may have very large values at the beginning. Therefore, we deploy a threshold $\theta_{a}$ to activate the fault detection mechanism after a short period at $t=T_{a}$, when $\operatorname{det}(\Gamma(t)) \geq \theta_{a} . T_{a}$ is adjustable by tuning $\bar{\omega}$ in terms of the converging speed of the kernels. 
Therefore, the afore-defined error (34) admits the bound

$$
\epsilon_{\zeta_{2}}(t) \leq\left|P^{-1} \Gamma(t)^{-1}\right|\left[\bar{\epsilon}_{\mu_{0}}, \bar{\epsilon}_{\mu_{1}}, \ldots, \bar{\epsilon}_{\mu_{n_{\xi}-1}}\right]^{\top} \leq \bar{\epsilon}_{\zeta_{2}} \in \mathbb{R}^{n_{\star}},
$$

$\forall t \geq T_{a}$, where

$$
\bar{\epsilon}_{\mu_{h}}=\sum_{i=0}^{n_{\star}-1} \bar{\xi}_{g, i, h}
$$

Consequently, the estimation error of the state is bounded by

$$
\left|\epsilon_{x}\right| \triangleq|\hat{x}(t)-x(t)| \leq\left|T_{2}\right| \bar{\epsilon}_{\zeta_{2}} \triangleq \bar{\epsilon}_{x}(t) .
$$

On the otherhand, by recalling (25), we can then define the uncertainty on the fault detection residual

$$
\epsilon_{r_{F D}}(t)=\left|\left[V_{F^{(1)}} \epsilon_{\zeta_{2}}\right](t)+A_{22}\left[V_{F} \epsilon_{\zeta_{2}}\right](t)+\left[V_{F} g_{2}\right](t)\right| .
$$

Notice that

$$
\begin{aligned}
{\left[V_{F} \kappa\right](t) } & \leq \bar{\kappa} \int_{0}^{t}|F(t, \tau)| d \tau \\
& =\bar{\kappa}\left[e^{-2 \omega t}\left(\frac{e^{\omega t}-1}{\omega}-\frac{e^{2 \omega t}-1}{2 \omega}\right)+e^{-\omega t}\left(\frac{e^{\omega t}-1}{\omega}-t\right)\right] \\
& \triangleq \bar{\chi}_{\kappa, 0}(t) .
\end{aligned}
$$

where $\kappa$ and $\bar{\kappa}$ represents the signals $\epsilon_{\zeta_{1}}, \epsilon_{\zeta_{2}}, u$ and their corresponding upper bounds. Furthermore, it holds that

$$
\begin{aligned}
{\left[V_{F^{(1)}} \kappa\right](t) } & \leq \bar{\kappa} \int_{0}^{t}\left|F^{(1)}(t, \tau)\right| d \tau \\
& =\bar{\kappa}\left(-\int_{0}^{\frac{\ln \left(1+e^{t}\right)}{2}} F^{(1)}(t, \tau) d \tau+\int_{\frac{\ln \left(1+e^{t}\right)}{2}}^{t} F^{(1)}(t, \tau) d \tau\right) \\
& =\bar{\kappa} \frac{\left(e^{-t}-1\right)^{2}}{2} \triangleq \bar{\chi}_{\kappa, 1}(t) .
\end{aligned}
$$

The above BIBO property of Volterra operators with BC-NK kernels leads to the following bounding inequalities:

$$
\left|\left[V_{F} \epsilon_{\zeta_{2}}\right](t)\right| \leq \bar{\chi}_{\zeta, 0}(t),\left|\left[V_{F} g_{2}\right](t)\right| \leq \bar{\chi}_{g_{2}, 0}(t),\left|\left[V_{F^{(1)}} \epsilon_{\zeta_{2}}\right](t)\right| \leq \bar{\chi}_{\zeta, 1}(t) .
$$

180

Then, the effects of the uncertainty on the fault detection residual satisfies

$$
\left\|\epsilon_{r_{F D}}(t)\right\| \leq\left\|\bar{\chi}_{\zeta, 1}(t)+\left|A_{22}\right| \bar{\chi}_{\zeta, 0}(t)+\bar{\chi}_{g_{2}, 0}(t)\right\| \triangleq \sigma_{F D}(t),
$$

which can be used as a threshold for fault detection. 
Proposition 5.1. A fault occurring in the system is detected by the proposed fault detection scheme at time $t=T_{D}$, once the fault detection residual $r_{F D}(t)$ exceeds the fault detection threshold $\sigma_{F D}$, i.e. $r_{F D}\left(T_{D}\right)>\sigma_{F D}\left(T_{D}\right)$.

Fault detectability If there exists a time instant $T_{D}>T_{0}$, such that the fault $f(t, u, y)$ fulfils the following inequality

$$
\left\|F_{f, 2}\left[V_{F} f\right]\left(T_{D}\right)\right\|>\left\|\bar{\chi}_{\zeta, 1}\left(T_{D}\right)+\left|A_{22}\right| \bar{\chi}_{\zeta, 0}\left(T_{D}\right)+\bar{\chi}_{g_{2}, 0}\left(T_{D}\right)\right\|,
$$
then it can be detected at $T_{D}$.

For the purpose of fault isolation, referring to 40 and (41), it is easy to show that the images computed based on the operator $\breve{V}_{F}$ are bounded by

$$
\left|\left[\breve{V}_{F} \epsilon_{\zeta_{2}}\right](t)\right| \leq \overline{\bar{\chi}}_{\zeta, 0}(t),\left|\left[V_{F} g_{2}\right](t)\right| \leq \overline{\tilde{\chi}}_{g_{2}, 0}(t),\left|\left[\breve{V}_{F^{(1)}} \epsilon_{\zeta_{2}}\right](t)\right| \leq \overline{\tilde{\chi}}_{\zeta, 1}(t),
$$

with

$$
\begin{aligned}
& {\left[\breve{V}_{F} \kappa\right](t) \leq \bar{\kappa} \int_{0}^{t-T_{D}}\left|F\left(t-T_{D}, \tau\right)\right| d \tau} \\
& =\bar{\kappa}\left[e^{-2 \omega\left(t-T_{D}\right)}\left(\frac{e^{\omega\left(t-T_{D}\right)}-1}{\omega}-\frac{e^{2 \omega\left(t-T_{D}\right)}-1}{2 \omega}\right)+e^{-\omega\left(t-T_{D}\right)}\left(\frac{e^{\omega\left(t-T_{D}\right)}-1}{\omega}-t+T_{D}\right)\right] \\
& \triangleq \bar{\chi}_{\kappa, 0}(t),
\end{aligned}
$$

and

$$
\begin{aligned}
& {\left[\breve{V}_{F^{(1)}} \kappa\right](t) \leq \bar{\kappa} \int_{0}^{t-T_{D}}\left|F^{(1)}\left(t-T_{D}, \tau\right)\right| d \tau} \\
& =\bar{\kappa}\left(-\int_{0}^{\frac{\ln \left(1+e^{\left.t-T_{D}\right)}\right.}{2}} F^{(1)}\left(t-T_{D}, \tau\right) d \tau+\int_{\frac{\ln \left(1+e^{\left.t-T_{D}\right)}\right.}{2}}^{t-T_{D}} F^{(1)}\left(t-T_{D}, \tau\right) d \tau\right) \\
& =\bar{\kappa} \frac{\left(e^{-\left(t-T_{D}\right)}-1\right)^{2}}{2} \triangleq \overline{\bar{\chi}}_{\kappa, 1}(t) .
\end{aligned}
$$

As such, upon the occurrence of the $i$-th fault, the $i$-th fault isolation residual defined in 30 verifies the following inequality

$$
\left\|\epsilon_{r_{F I, i}}(t)\right\| \leq\left\|\overline{\bar{\chi}}_{\zeta, 1}(t)+\left|A_{22}\right| \overline{\bar{\chi}}_{\zeta, 0}(t)+\overline{\bar{\chi}}_{g_{2}, 0}(t)\right\| \triangleq \sigma_{F I}(t), \forall t>T_{D}
$$

giving rise to the following fault isolation logic.

Proposition 5.2. By using the fault isolation residual $r_{F I, i}(t)$ in (30) and the fault isolation threshold $\sigma_{F I, i}(t)$ in (46), the $p$-th fault is excluded at $T_{E, p}$, if $r_{F I, p}(t)$ exceeds the corresponding threshold, i.e. $\quad \exists T_{E, p} \geq T_{D}$ such that $r_{F I, p}\left(T_{E, p}\right)>\sigma_{F I}\left(T_{E, p}\right)$. If all the faults are excluded except $q$-th fault, i.e. $\left(\exists T_{D}<t_{2, i}<T_{I}, r_{F I, i}\left(t_{2, i}\right)>\sigma_{F I}\left(t_{2, i}\right), \forall i \in\left\{0, \ldots, N_{\mathcal{F}}-1\right\} \backslash q\right) \wedge\left(r_{F I, q} \leq\right.$ $\left.\sigma_{F I, q}(t), \forall t>T_{D}\right)$, then the $q$-th fault is isolated. 
Fault isolability Given a fault $\phi_{q} \in \mathcal{F}$, if for each $i \in\left\{0, \ldots, N_{\mathcal{F}}-1\right\} \backslash q$, there exists some $t_{2, i}>T_{D}$ such that the mismatch between the $q$-th fault and $i$-th fault verifies the following condition

$$
\left\|F_{f, 2}\left|\left[\breve{V}_{F} \phi_{q}\right]\left(t_{2, i}\right)-\left[\breve{V}_{F} \phi_{i}\right]\left(t_{2, i}\right)\right|\right\|>\left\|\overline{\tilde{\chi}}_{\zeta, 1}\left(t_{2, i}\right)+\left|A_{22}\right| \overline{\tilde{\chi}}_{\zeta, 0}\left(t_{2, i}\right)+\overline{\widetilde{\chi}}_{g_{2}, 0}\left(t_{2, i}\right)\right\|,
$$

then the $q$-th fault will be isolated at time $T_{I}=\underset{i \in\left\{0, \ldots, N_{\mathcal{F}}-1\right\} \backslash q}{\max } t_{2, i}$.

Remark 5.2. Recalling the Volterra transformation systems (20) and (27), the eters $\omega_{h}, \omega$ act as the forgetting factors in the transformations. Therefore, increasing the parameters is prone to make the fault detection scheme more sensitive to the occurrence of the fault. However, Equations (36), (40) and (41) together imply that larger values of the parameters will sacrifice the tightness of the thresholds especially in the initial phase, when $\Gamma(t)$ are small. As such the detectability of the faults at the beginning could be degraded. Therefore, a compromise is necessary to deal with the trade-off between the sensitivity of the FDI response and the detectability of fast-occurring faults. The interested readers can get a deeper insight into detailed numerical analysis concerning parameter tuning, kernel features and computational complexity of the kernel-based estimators in [35].

To summarize, the overall FDI scheme can be concluded as in Fig. 1

\section{Numerical Example}

In this section, we consider the dynamic model of an accumulator in a web processing line introduced in [38. The schematic of the carriage, the web spans and the rollers within an accumulator are illustrated in Fig. 2. The number of the rollers on the carriage is assumed to be $N / 2$ and $N+1$ rollers in total are present in the accumulator. As a result, the $j$-th roller dynamic can be modelled as

$$
\begin{aligned}
\dot{t}_{j}(t) & =\frac{A_{a} E_{e} R}{x_{c}}\left(\omega_{j}(t)-\omega_{j-1}(t)\right)+\frac{R}{x_{c}}\left[t_{j-1}(t) \omega_{j-1}(t)-t_{j}(t) \omega_{j}(t)\right] \\
J \dot{\omega}_{j}(t) & =-B_{j} \omega_{j}(t)+R\left(t_{j+1}(t)-t_{j}(t)\right)
\end{aligned}
$$

where $j \in\{1, \ldots, N\}, A_{a}$ denotes the area of the cross section of the strip. $B_{j}$ 


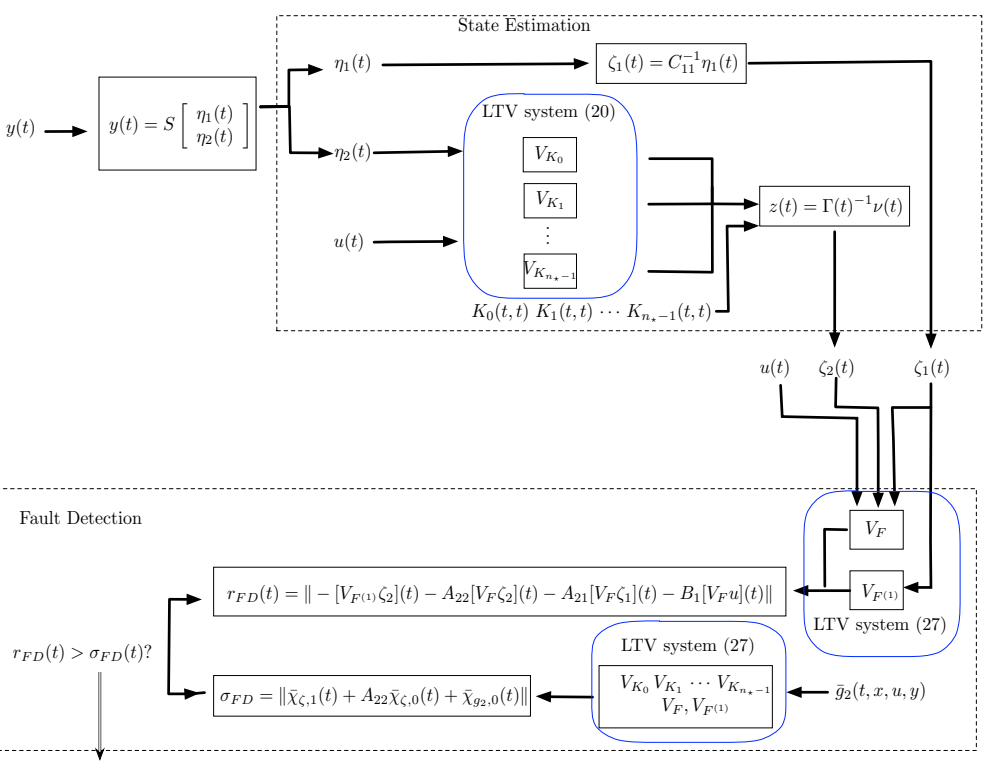

Fault Detection Decision $t=T_{D}$

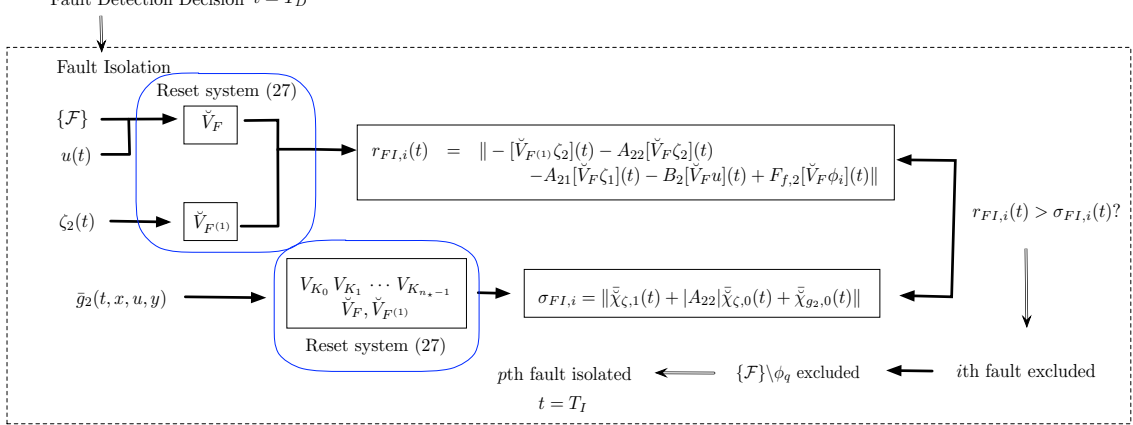

Figure 1: Flowchart of the fault detection and isolation scheme.

material. $J$ is the moment of inertia of the roller and $R$ represents the radius of the roller. $t_{j}(t), \omega_{j}(t)$ denotes the tension and the angular velocity of the $j$-th roller, respectively.

By defining the averaging tension $t_{c}=\frac{1}{N} \sum_{j=1}^{N} t_{j}(t)$ and considering the driven roller angular dynamics at both sides of the accumulator, the dynamics of this process can be formally expressed as

$$
\left\{\begin{array}{l}
\dot{x}(t)=A x(t)+B u(t)+E g_{U}(t, x, u) \\
y(t)=C x(t)
\end{array}\right.
$$




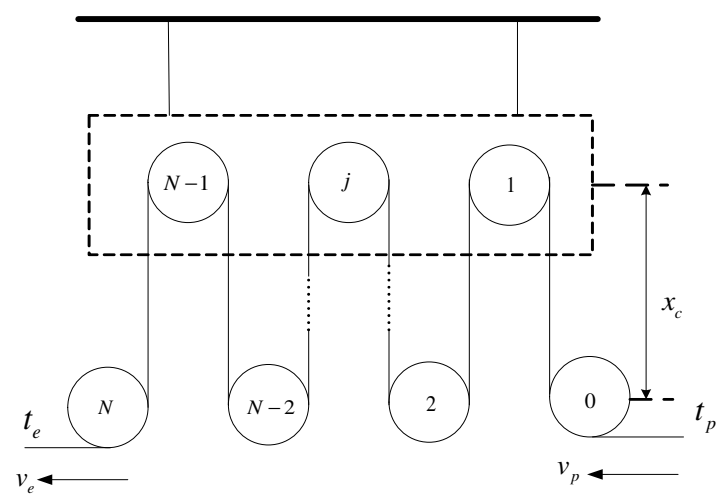

Figure 2: Sketch of an accumulator in the web processing line.

with

$$
\begin{gathered}
x(t)=\left[t_{c}(t), v_{e}(t), v_{p}(t)\right]^{\top}, A=\left[\begin{array}{ccc}
0 & \frac{A_{a} E_{e}}{x_{c} N} & -\frac{A_{a} E_{e}}{x_{c} N} \\
-\frac{R^{2}}{J} & -\frac{B_{f e}}{J} & 0 \\
-\frac{R^{2}}{J} & 0 & -\frac{B_{f p}}{J}
\end{array}\right], \\
B=\left[\begin{array}{ccc}
0 & 0 & 0 \\
\frac{R K_{e}}{J} & 0 & \frac{R^{2}}{J} \\
0 & \frac{R K_{p}}{J} & -\frac{R^{2}}{J}
\end{array}\right], \quad C=\left[\begin{array}{lll}
0 & 1 & 0 \\
0 & 0 & 1
\end{array}\right], \\
E=\left[\begin{array}{ccc}
0 & \frac{R^{2}}{J} & 0
\end{array}\right]^{\top}, u(t)=\left[u_{e}(t), u_{p}(t), t_{r}\right],
\end{gathered}
$$

As in [38, it is reasonable to assume that the tensions $t_{j}(t), \forall j \in\{1, \ldots, N\}$ are negligible compared to the product $A_{a} E_{e} . \quad v_{e}(t)=R \omega_{N}(t)$ and $v_{p}(t)=$ $R \omega_{0}(t)$ are the strip velocities and the process and exit end respectively. Assume $t_{p}(t)$ is maintained close to a desired web tension $t_{r}$ and $t_{e}(t)$ is defined as $t_{e}=t_{r}+g_{U}(t, x, u)$ where $g_{U}(t, x, u)$ is a unknown non-linear function depending on the complicated relationship among each rollers, which is hard to be characterized explicitly.

To regulate the stripe velocities $v_{e}(t), v_{p}(t)$ with respect to the desired values $v_{r, e}$ and $v_{r, p}$, a PID controller is applied providing control signals $u_{e}(t)$ and $u_{p}(t)$.

$$
\begin{aligned}
& u_{e}(t)=\frac{J}{P K_{e}}\left(\frac{B_{f e}}{J} v_{r, e}-k_{p e} e_{v e}(t)-k_{i e} \int_{0}^{t} e_{v e}(\tau) d \tau\right), \\
& u_{p}(t)=\frac{J}{P K_{p}}\left(\frac{B_{f p}}{J} v_{r, p}-k_{p p} e_{v p}(t)-k_{i p} \int_{0}^{t} e_{v p}(\tau) d \tau\right),
\end{aligned}
$$

where

$$
e_{v e}(t) \triangleq v_{e}(t)-v_{r, e}, \quad e_{v p} \triangleq v_{p}(t)-v_{r, p} .
$$


In the simulation, we adopt the same values as in [38, $A_{a}=3.27 \times 10^{-4} \mathrm{~m}^{2}, E_{e}=$ $6.90 \times 10^{10}, N=34, v_{f}=35.037 \times 10^{5} \mathrm{~N} \cdot \mathrm{s} / \mathrm{m}, R=0.1524 \mathrm{~m}, \mathrm{~J}=2.1542 \mathrm{~kg} \cdot \mathrm{m}^{2}$, $B_{f e}=0.018$ and $B_{f p}=0.022 . x(0)=\left[x_{0}(0), \ldots, x_{3}(0)\right]^{\top}=\left[\begin{array}{lll}5170 & 2 & 2\end{array}\right]^{\top}$. The controller are tuned by $k_{p e}=k_{p p}=40$ and $k_{i e}=k_{i p}=5$. The desired tension $t_{r}=5180 \mathrm{~N}$ and the desired speed are set to $v_{r}=3.3 \mathrm{~m} / \mathrm{s}$.

We assume there is a drifting fault occur on the actuator on the process side of the accumulator such that $u_{p, f}(t)=u_{p}(t)+f(t)$ with $f(t)=\mathcal{B}(t-3) 10 t$. Two typical kinds of actuator faults are assumed to be included in the fault set

$$
\mathcal{F}=\left\{\phi_{1}(t)=\left[\begin{array}{lll}
0 & 10 t & 0
\end{array}\right]^{\top}, \phi_{2}=\left[\begin{array}{lll}
0 & 10 & 0
\end{array}\right]^{\top}\right\},
$$

where $\phi_{1}(t)$ is the drifting fault and $\phi_{2}(t)$ denotes the bias fault.

The simulation is implemented in the Matlab/Simulink with 4-th order Runge-Kutta solver and the sampling interval of $T_{s}=10^{-3} \mathrm{~s}$. The proposed scheme is parametrized by $\bar{\omega}=2.5, \omega_{1}=10, \omega_{2}=20, \omega=1, \theta_{a}=0.24$.

Based on the formulated web process system, the simulation is conducted considering two scenarios.

\subsection{Fixed carriage}

In this scenario, we assume that the position of the carriage is fixed, i.e. $x_{c}=5 m$.

235 Making use of the proposed finite-time observer in Section 4 , the state estimation results are illustrated in Fig. 3 Moreover, the state estimation results are compared with the results of an SM method proposed in [39, tuned by $\eta_{1}=10, \eta_{2}=2, \eta_{3}=1, \epsilon=0.01$ and $\alpha_{m}=0.1$.

It is readily seen that before the occurrence of the drifting fault $\phi_{1}(t)$ at $t=3 \mathrm{~s}$, the kernel-based state estimates converge instantaneously to the true state variables regardless of the unknown non-linearity $g_{U}(t, x, u)$. However, the SMC observer is vulnerable to the uncertainty and the estimates fluctuated continuously. The state estimation error of the SMC method undergoes a converging process and exists persistently. Therefore, it would be difficult for such estimates to contribute to prompt FDI. On the other hand, the computational complexity of both methods are compared in Table. 1 by the number of computation flops (NCF) per sampling step and the algorithm elapsed time (AET). The table shows that the SMC method has advantages in terms of computational simplicity.

Table 1: Comparison of computational complexity of both methods

\begin{tabular}{|c|c|c|}
\hline Methods & kernel-based methods & SMC method \\
\hline NCF & 237 & 53 \\
\hline AET & $8.53 s$ & $3.56 s$ \\
\hline
\end{tabular}



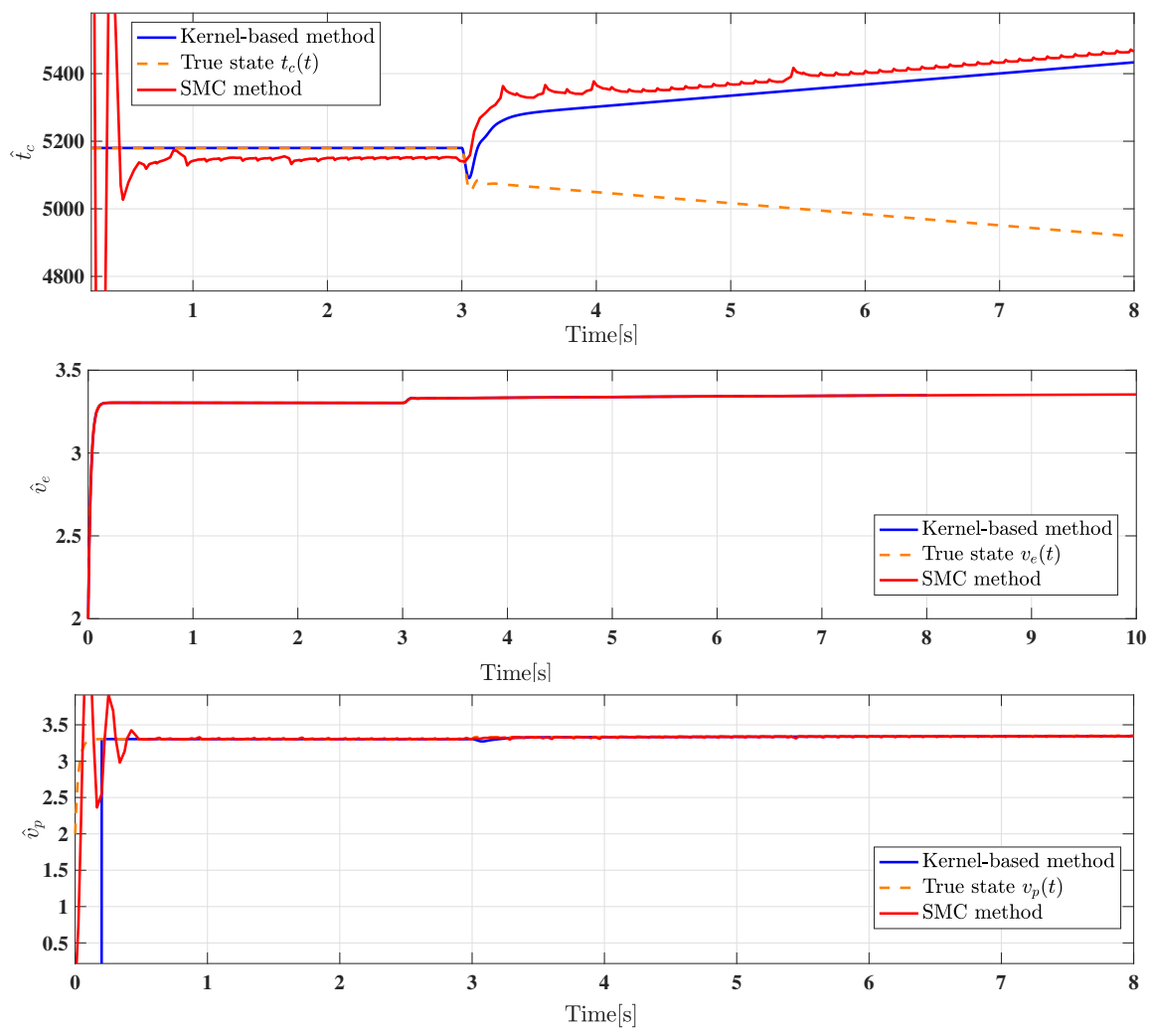

Figure 3: State estimation of the web process system with fixed carriage.

The proposed FDI scheme can be attained by the FDI residuals as shown in Fig. 4. Thanks to the deadbeat convergence feature, the fault detection residual differs from 0 immediately after the occurrence of fault at $t=3 \mathrm{~s}$. Subsequent activation of the fault isolation scheme gives the rapid growth of $r_{F I, 2}(t)$, thus excluding $\phi_{2}(t)$ and isolating $\phi_{1}(t)$, i.e. the drifting fault of the actuator on the processing side.

\subsection{Suspended carriage}

Due to practical needs, the position of the carriage may need to be changed. Therefore, suspended carriages are usually adopted catering to different processing requirements. As a result, the suspension is likely to introduce an uncertainty on $x_{c}$ that influences the dynamics of $t_{c}(t)$ that influencing the output $y(t)$ in an indirect way. However, due to the physical space limitation, the uncertainty on $x_{c}$ is bounded. This scenario is described by the problem formulation of the FDI scheme proposed in Section 5. We assume the carriage undergoes a periodic swing, i.e. $x_{c}(t)=x_{n, c}+\Delta x_{c}(t)$, such that $x_{n, c}=5 m$ and 

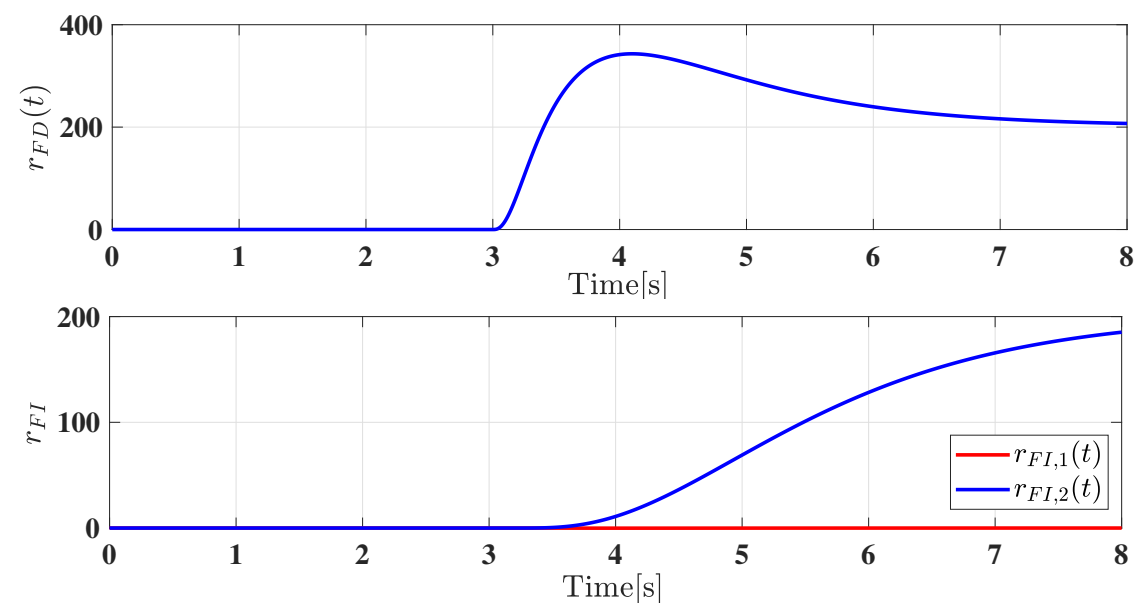

Figure 4: Fault detection and isolation residuals of the web process system with fixed carriage.

$\Delta x_{c}(t)=\sin (0.2 t) m$. Therefore, system 50 can be reformulated as

$$
\left\{\begin{array}{l}
\dot{x}(t)=A x(t)+B u(t)+g_{L}(t, y)+E g_{U}(t, x, u) \\
y(t)=C x(t)
\end{array}\right.
$$

where $g_{L}(t, y)=\left[\begin{array}{lll}g_{t c}(t) & 0 & 0\end{array}\right]^{\top}, g_{t c}(t)=-\frac{A_{a} E_{e} \Delta x_{c}(t)}{x_{c}(t) x_{n, c} N}\left[\begin{array}{ll}1 & -1\end{array}\right] y(t)$. A deterministic bound can be calculated for the unknown non-linearity as

$$
\left|g_{t c}(t)\right| \leq\left|\frac{A_{a} E_{e}}{\left(x_{n, c}-1\right) x_{n, c} N}[1-1] y(t)\right|=3.181 \times 10^{4}|[1-1] y(t)| \triangleq \bar{g}_{t c}(t) .
$$

Correspondingly, thresholds can be computed based on $\bar{g}_{t c}(t)$. This allows to achieve fault detection and isolation decisions as shown in Fig. 5.

As the fault occurs at $t=3 \mathrm{~s}$, the fault detection residual increases immediately and crosses the fault detection threshold at $t=3.344 \mathrm{~s}$; the fault isolation is achieved by excluding $\phi_{2}(t, y, u)$ at $t=4.166 \mathrm{~s}$.

It is worth noting that $F_{f, 2}$ is not full column rank, so 24 is not sensitive to the fault on the exit end. However, referring to [38, it is reasonable to assume either $t_{e}(t)$ or $t_{p}(t)$ to be close to $t_{r}$. As a result, by denoting $t_{p}=$ $t_{r}+g_{U}(t, x, u)$ and correspondingly changing $E=\left[0,0, \frac{-R^{2}}{J}\right]^{\top}$ in 50 , a similar fault diagnosor can established for such problem formulation to detect the fault on $u_{e}(t)$. Fig. 6 confirms that the fault detection threshold for the fault on the processing end is not sensitive to the fault on the exit side. Therefore, by constructing two parallel FDI schemes for $u_{e}(t)$ and $u_{p}(t)$, the health status on both actuators can be monitored independently. 

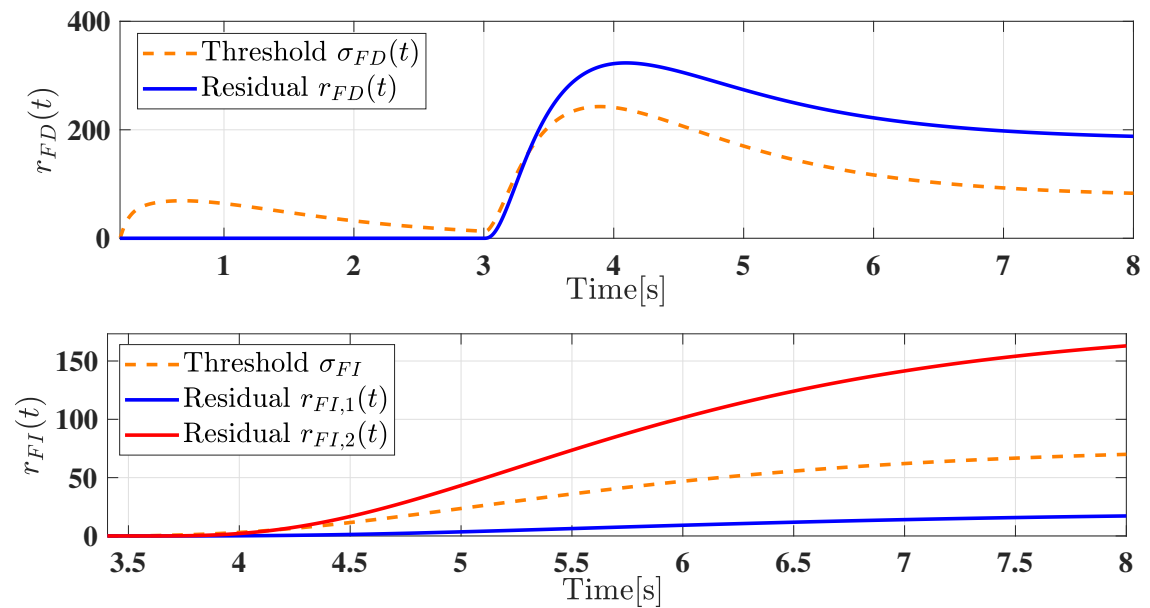

Figure 5: Fault detection and isolation of the web process system with suspended carriage.

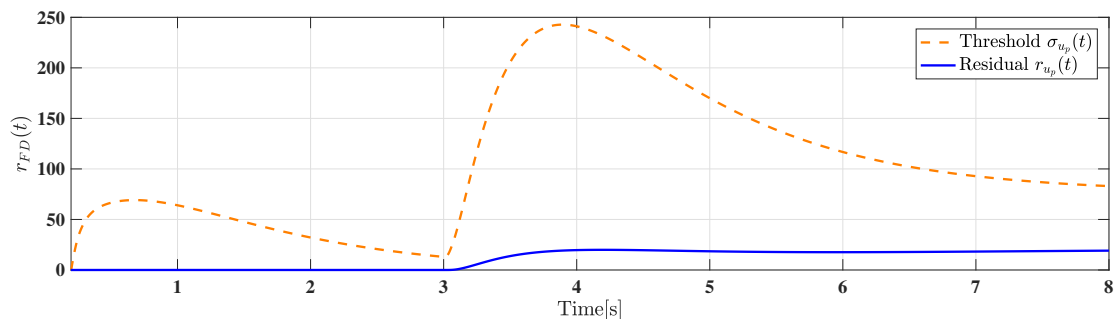

Figure 6: Fault detection residual and threshold on the processing side when the fault occurs to the actuator on the exit side.

\section{Concluding Remarks}

In this paper, a fast-convergent FDI scheme is designed for a class of systems affected by unknown uncertainties or unbounded nonlinearities. Making use of system decomposition, such uncertain systems can be transformed into linear systems with and without the intractable uncertainty, respectively. As a result, the non-asymptotic Volterra operator is applicable, so that finite-time convergence can be achieved for state estimation. Moreover, based on the deadbeat state estimates, once the fault occurs, it can be detected and isolated in an efficient way. An application example has been considered to address the problem of the actuator faults detection and isolation in a web process system. The effectiveness of the proposed FDI schemes has been confirmed in two scenarios.

\section{Acknowledgment}

We would like to acknowledge Prof. Thomas Parisini for many fruitful research discussions and for help and suggestions on fault diagnosis architectures. 


\section{References}

[1] R. J. Patton, P. M. Frank, R. N. Clarke (Eds.), Fault Diagnosis in Dynamic Systems: Theory and Application, Prentice-Hall, Inc., 1989.

[2] M. Blanke, M. Kinnaert, J. Lunze, M. Staroswiecki, J. Schröder, Diagnosis and fault-tolerant control, Vol. 2, Springer, 2006.

[3] J. Gertler, Fault Detection and Diagnosis, Springer, 2015.

[4] R. Isermann, Process fault detection based on modeling and estimation methods - a survey, Automatica 20 (4) (1984) 387-404.

[5] P. M. Frank, X. Ding, Survey of robust residual generation and evaluation methods in observer-based fault detection systems, Journal of process control 7 (6) (1997) 403-424.

[6] X. He, Z. Wang, Y. Liu, D. H. Zhou, Least-squares fault detection and diagnosis for networked sensing systems using a direct state estimation approach, IEEE Transactions on Industrial Informatics 9 (3) (2013) 16701679 .

[7] D. M. Raimondo, F. Boem, A. Gallo, T. Parisini, A decentralized faulttolerant control scheme based on active fault diagnosis, in: 2016 IEEE 55th Conference on Decision and Control (CDC), IEEE, 2016, pp. 2164-2169.

[8] A. Vemuri, M. M. Polycarpou, On-line approximation methods for robust fault detection, in: Proc. 13th IFAC World Congress, no. 319-324, 1996.

[9] R. M. Ferrari, T. Parisini, M. M. Polycarpou, Distributed fault detection and isolation of large-scale discrete-time nonlinear systems: An adaptive approximation approach, IEEE Transactions on Automatic Control 57 (2) (2012) 275-290.

[10] F. Boem, C. Keliris, T. Parisini, M. M. Polycarpou, Fault diagnosis for uncertain networked systems, in: Uncertainty in Complex Networked Systems, Springer, 2018, pp. 533-581.

[11] X. Zhang, T. Parisini, M. M. Polycarpou, Adaptive fault-tolerant control of nonlinear uncertain systems: an information-based diagnostic approach, IEEE Transactions on Automatic Control 49 (8) (2004) 1259-1274.

[12] V. Reppa, M. M. Polycarpou, C. G. Panayiotou, Adaptive approximation for multiple sensor fault detection and isolation of nonlinear uncertain systems, IEEE Transactions on Neural Networks and Learning Systems 25 (1) (2014) 137-153.

[13] X. Liu, X. Gao, J. Han, Observer-based fault detection for high-order nonlinear multi-agent systems, Journal of the Franklin Institute 353 (1) (2016) 72-94. 
[14] J. Zhang, A. K. Swain, S. K. Nguang, Robust sensor fault estimation scheme for satellite attitude control systems, Journal of the Franklin Institute 350 (9) (2013) 2581-2604.

[15] W. Chen, W.-T. Chen, M. Saif, M.-F. Li, H. Wu, Simultaneous fault isolation and estimation of lithium-ion batteries via synthesized design of luenberger and learning observers, IEEE Transactions on Control Systems Technology 22 (1) (2014) 290-298.

[16] Y. Shtessel, C. Edwards, L. Fridman, A. Levant, Introduction: Intuitive Theory of Sliding Mode Control, 2014.

[17] S. K. Spurgeon, Sliding mode observers: a survey, International Journal of Systems Science 39 (8) (2008) 751-764.

[18] D.-y. Liu, O. Gibaru, W. Perruquetti, Parameters estimation of a noisy sinusoidal signal with time-varying amplitude, in: 2011 19th Mediterranean Conference on Control \&amp; Automation (MED), IEEE, 2011, pp. 570575 .

[19] H. Sira-Ramirez, Dynamic second-order sliding mode control of the hovercraft vessel, Control Systems Technology IEEE Transactions on 10 (6) (2002) 860-865.

[20] A. Levant, Homogeneity approach to high-order sliding mode design , Automatica 41 (5) (2005) 823-830.

[21] A. Polyakov, A. Poznyak, Lyapunov function design for finite-time convergence analysis of "twisting" and "super-twisting" second order sliding mode controllers, in: International Workshop on Variable Structure Systems, 2008.

[22] M. Fliess, H. Rez, An algebraic framework for linear identification, Esaim Control Optimisation \& Calculus of Variations 9 (9) (2010) 151-168.

[23] Michel, Cedric, SIRARAMIREZ, Hebertt, Robust residual generation for linear fault diagnosis: an algebraic setting with examples, International Journal of Control 77 (14) (2004) 1223-1242.

[24] G. Fedele, L. Coluccio, A recursive scheme for frequency estimation using the modulating functions method, Applied Mathematics \& Computation 216 (5) (2010) 1393-1400.

[25] D.-Y. Liu, T.-M. Laleg-Kirati, W. Perruquetti, O. Gibaru, Non-asymptotic state estimation for a class of linear time-varying systems with unknown inputs, IFAC Proceedings Volumes 47 (3) (2014) 3732-3738.

[26] J. Jouffroy, J. Reger, Finite-time simultaneous parameter and state estimation using modulating functions, in: Control Applications, 2015. 
[39] M. Defoort, K. C. Veluvolu, J. J. Rath, M. Djemai, Adaptive sensor and actuator fault estimation for a class of uncertain lipschitz nonlinear systems, International Journal of Adaptive Control and Signal Processing 30 (2) (2016) 271-283. 